\title{
Télescope
}

Revue d'analyse comparée en administration publique

\section{Trois modèles de gouvernance multiniveau au-delà du clivage État-société}

\section{Jefferey M. Sellers}

Volume 19, numéro 1, hiver 2013

La gouvernance multiniveau

URI : https://id.erudit.org/iderudit/1017152ar

DOI : https://doi.org/10.7202/1017152ar

Aller au sommaire du numéro

Éditeur(s)

L'Observatoire de l'administration publique

ISSN

1929-3348 (numérique)

Découvrir la revue

Citer cet article

Sellers, J. M. (2013). Trois modèles de gouvernance multiniveau au-delà du clivage État-société. Télescope, 19(1), 62-84. https://doi.org/10.7202/1017152ar

\section{Résumé de l'article}

Par l'étude des interactions entre les institutions étatiques multiniveaux et l'organisation de la société civile, nous posons les jalons d'une plus grande compréhension de la gouvernance multiniveau. Les autorités locales et celles des échelons supérieurs créent des modèles de participation locale dans la vie politique et civique. Ces institutions évoluent à leur tour en fonction des modèles de pouvoir et d'influence au niveau local. À l'intérieur d'une démocratie bien établie, ces configurations institutionnelles et ces associations s'inscrivent dans une infrastructure interreliée qui dicte les conditions en matière de résolution collective de problèmes, de relations de pouvoir et de participation. Les complémentarités institutionnelles entre les formes d'autorités locales et les types d'organisations civiques viennent renforcer ces systèmes. Ces différences expliquent une grande partie des contrastes transnationaux dans l'élaboration des politiques et les démocraties locales. 


\title{
TROIS MODÈLES DE GOUVERNANCE MULTINIVEAU AU-DELÀ DU CLIVAGE ÉTAT-SOCIÉTÉ
}

\author{
Par Jefferey M. Sellers, Professeur, Department of Political Science, University of \\ Southern California, États-Unis•sellers@usc.edu \\ Traduit de l'anglais
}

\begin{abstract}
RÉSUMÉ Par l'étude des interactions entre les institutions étatiques multiniveaux et l'organisation de la société civile, nous posons les jalons d'une plus grande compréhension de la gouvernance multiniveau. Les autorités locales et celles des échelons supérieurs créent des modèles de participation locale dans la vie politique et civique. Ces institutions évoluent à leur tour en fonction des modèles de pouvoir et d'influence au niveau local. À l'intérieur d'une démocratie bien établie, ces configurations institutionnelles et ces associations s'inscrivent dans une infrastructure interreliée qui dicte les conditions en matière de résolution collective de problèmes, de relations de pouvoir et de participation. Les complémentarités institutionnelles entre les formes d'autorités locales et les types d'organisations civiques viennent renforcer ces systèmes. Ces différences expliquent une grande partie des contrastes transnationaux dans l'élaboration des politiques et les démocraties locales.
\end{abstract}

\begin{abstract}
This paper, by exploring the interplay between multilevel state institutions and the organization of civil society, seeks to lay the groundwork for a deeper understanding of multilevel governance. Institutions within the local state and at higher levels systematically shape patterns of local participation in politics and civic life. In turn, these institutions evolve in ways that patterns of power and influence at the local level help to shape. In settled democracies, these configurations of institutions and associations comprise an interrelated infrastructure that sets terms for collective problem-solving, power relations and participation. Institutional complementarities between forms of local states and patterns of civic organization have reinforced these systems. These differences account for major crossnational contrasts in policy-making and local democracy.
\end{abstract}

Pour citer cet article : Sellers, J. M. (2013). « Trois modèles de gouvernance multiniveau au-delà du clivage État-société », Télescope, vol. 19, n 1, p. 62-84.

T a littérature portant sur la gouvernance multiniveau a montré la nécessité $\mathcal{L}$ de subdiviser l'État afin de mieux saisir les relations existant entre ses parties constituantes. Dans cet article, nous estimons qu'une approche segmentée s'avère nécessaire à l'analyse des relations de la société civile avec un État à paliers multiples. La division de la société et de l'État nous permet de mieux comprendre leurs relations - une thématique récurrente dans l'étude de la gouvernance - et par le fait même de dégager un meilleur portrait de la gouvernance multiniveau. Notre étude se penche sur les liens entre l'organisation sociétale et l'État à une échelle locale, soit là où s'opère en grande partie la gouvernance multiniveau. Ces relations se tissent au sein d'une infrastructure modelée par les institutions étatiques, par les autorités locales de même que par les schèmes organisationnels au sein de la société civile, et ce, autant à l'échelle locale que supralocale. Les complémentarités institutionnelles dans l'organisation de la société civile et de l'État ont généré 
trois différents modèles des relations entre l'État et la société. Ces modèles mettent en évidence les variations dans les dynamiques de gouvernance multiniveau au sein de démocraties bien établies.

L'attention portée par la littérature sur les accords impliquant l'échelle locale indique l'importance de la politique et de la gouvernance sur ce plan. Les systèmes de gouvernance locale - soit ceux se situant du côté de la ville ou de la communauté - représentent une caractéristique des démocraties contemporaines depuis l'émergence de l'État-nation au XIXe siècle. Les démocraties plus anciennes présentent généralement des instances très institutionnalisées en matière de prise de décision, de mise en œuvre des politiques et de participation politique. Ces institutions, souvent ancrées dans les garanties constitutionnelles, émanent de traditions de longue date (Hesse, 1991; Lidström, 2003; Page et Goldsmith, 1989; Pierre, 1999; Vetter, 2007). L'autonomie fréquente des gouvernements locaux devrait en soi être suffisante pour justifier des analyses comparatives à l'échelle locale. Les institutions et les agents locaux et régionaux sont des éléments essentiels à la politique, tel qu'il a été illustré par de récentes études émanant de plusieurs perspectives (John et Cole, 2000; Ostrom, 1990; Putnam, Leonardi et Nanetti, 1993; Sabel et autres, 1989; Savitch, Kantor et Vicari, 2002; Sellers, 2002). Les cadres d'analyse qui tiennent compte de ces variations infranationales montrent que les processus et les conditions à l'échelle locale et régionale font une différence sur les plans du capital social (Putnam, Leonardi et Nanetti, 1993), de l'engagement civique (Varshney, 2002) ou de la productivité économique (Herrigel, 1996).

Les communautés locales, en tant que sociétés et entités politiques locales, sont également des lieux où se déroule la politique. L'organisation des partis et des mouvements politiques se fait souvent à l'échelle des communautés et des quartiers. Les organisations économiques telles que les entreprises et les syndicats organisent les milieux de travail des usines de fabrication, des bureaux et des magasins. Des associations civiques, que ce soit des organismes bénévoles de tous types ou des associations de quartier, ancrent leurs actions dans la communauté. Une analyse de la gouvernance multiniveau qui s'appuie uniquement sur l'État ne peut rendre compte de l'apport de ces organisations sociétales à la prise de décision politique et à la gouvernance. De nouveaux modèles des relations multiniveaux entre l'État et la société civile illustrent l'action de ces forces sociales dans la politique.

Notre article repose sur des comparaisons empiriques systématiques de ces mécanismes de gouvernance locale dans quarante-quatre pays (Sellers, Lidström et Bae, à paraître). Nous relevons trois configurations parallèles qui caractérisent les institutions de gouvernance locale des pays développés. Des configurations similaires apparaissent également au sein des pays en transition où se tient un processus de construction étatique et de renforcement de la démocratie. 


\section{- UNE APPROCHE À NIVEAUX MULTIPLES POUR COMPRENDRE LES RELATIONS ENTRE L'ÉTAT ET LA SOCIÉTÉ}

Nous nous appuyons sur une approche à niveaux multiples des hiérarchies au sein de l'État et de la société pour comprendre les processus locaux et leurs interactions à l'échelle nationale. La stratification, ou la superposition, de systèmes institutionnels demeure un aspect fondamental de la diversité institutionnelle des démocraties développées (Schickler, 2001; Thelen, 2004). Les hiérarchies territoriales des États-nations illustrent un exemple universel de cette stratification. Une analyse de ces hiérarchies locales nécessite le renversement de l'image traditionnelle descendante des hiérarchies. Cette approche éclaire sur l'ensemble des processus nationaux et locaux demeurés invisibles ou obscurs dans les études transnationales des relations entre l'État et la société.

Bien que les plus hauts ordres de gouvernement de l'État demeurent une présence constante de l'analyse, un portrait stratifié apporte un éclairage neuf quant à leurs rôles. Les gouvernements locaux servent l'intérêt de l'État dans ce que Mann a qualifié de "puissance infrastructurelle " (infrastructural power) (Mann, 1984; Soifer, 2008). Ils fournissent le personnel organisationnel et la présence physique pour mener à terme les objectifs de l'État au sein des communautés. Parallèlement à cette capacité fonctionnelle, ils tentent de renforcer le soutien pour l'autorité de l'État dans la communauté. Par ailleurs, l'appui au local s'avère essentiel au maintien de la puissance nationale pour les élites d'États-nations démocratiques qui dépendent du soutien électoral. Dans les États-nations démocratiques du XIX siècle et du début du XXe siècle en Europe et en Amérique du Nord, la gouvernance locale se résumait à ce que faisaient les structures étatiques plus larges. Avec l'émergence d'États nationaux dotés de programmes liés à l'aide sociale, au développement économique et à la qualité de la vie, le rôle des gouvernements nationaux et intermédiaires s'est étendu dans la majorité de ces pays.

Les travaux portant sur les gouvernements locaux signalent la participation de ces derniers à divers degrés et de différentes façons. Leur façon de procéder résulte de leur pouvoir formel de même que des ressources financières mises à leur disposition. Outre ces facteurs, la mobilisation croissante au sein des entreprises et des militants locaux et la diffusion d'une gouvernance active au sein des autorités locales ont contribué à la montée de la gouvernance locale. Cette mobilisation locale rend de plus en plus difficile la délégation des problématiques locales vers des gestionnaires étatiques ou des directeurs hiérarchiques ou la considération des politiques locales simplement en matière de relations avec le " centre » national. Pour rendre compte de ses possibilités, la gouvernance locale doit être considérée comme une arène liée à plusieurs autres.

La société civile et les organisations politiques jouent également un rôle au sein des localités et dans les arènes régionale et nationale. Quant aux partis et aux mouvements sociaux, l'organisation locale compte généralement un élément lié à la construction d'organisations nationales. D'autres mouvements peuvent se concentrer sur des communautés individuelles ou encore déployer leurs efforts de mobilisation en fonction de l'ordre de gouvernement ciblé (Tarrow, 2005). Des groupes comme le Sierra Club aux États-Unis mobilisent la population autour de 
questions nationales à l'aide d'organisations locales. D'autres, tel le mouvement antinucléaire allemand, transposent des controverses locales en mobilisations à grande échelle. La possibilité du niveau local de participer à l'élaboration d'une politique des échelons étatiques institutionnels vient influencer ces choix (Skocpol, 2003).

En traçant le portrait de cette réalité des institutions gouvernementales et de la société civile, l'approche à niveaux multiples soulève de nouvelles questions sur les façons dont les politiques locales se rattachent aux politiques de plus haut niveau et sur la manière de penser les relations État-société globalement. Les études antérieures ayant examiné les relations " centrales-locales " privilégiaient les relations des localités avec le centre et se concentraient généralement sur les institutions et les politiques officielles venant du sommet. Pour un pays, la représentation stratifiée de la gouvernance et des relations État-société au niveau local incarne également une composante essentielle. Les dynamiques d'échelle au sein de la société civile et de l'économie politique posent des questions parallèles et simultanées à celles à l'intérieur des institutions. Ce qui se produit dans la société au niveau local découle à la fois des institutions nationales et des organisations sociétales de même que de l'utilisation par les acteurs locaux des arrangements nationaux. De même, ce qui se produit au niveau national n'est qu'en partie attribuable à ce qui est communément appelée la " haute politique ». C'est également le résultat cumulé des structures organisationnelles et de l'identification des intérêts trop souvent relégués à la catégorie de basse politique. Une approche décentralisée s'avère nécessaire pour faire ressortir les modèles de gouvernance et des relations État-société (Sellers, 2005).

Une approche traditionnelle d'un État hiérarchique analyse les composants locaux en tant qu'élément d'une organisation plus large. L'analyse des liens locaux sous-tend la division du niveau local de chacune des institutions des autres niveaux. Cette opération permet d'analyser et de comparer différents modèles de relations : entre les organisations, les institutions et les autres acteurs à l'échelle locale; entre les autorités locales et les autorités d'autres ordres de gouvernement; entre les autres acteurs et institutions à l'échelle locale et nationale. L'objectif est de dégager une nouvelle vision des relations État-société à l'échelle locale et de la relation entre la politique de l'État et de la société à des niveaux plus élevés. Au moment où cette conceptualisation désagrège les hiérarchies territoriales au sein de la société et de l'État, elle désagrège également les secteurs de la société et de l'État (figure 1). 


\author{
RELATIONS GLOBALES (MACRO)
}

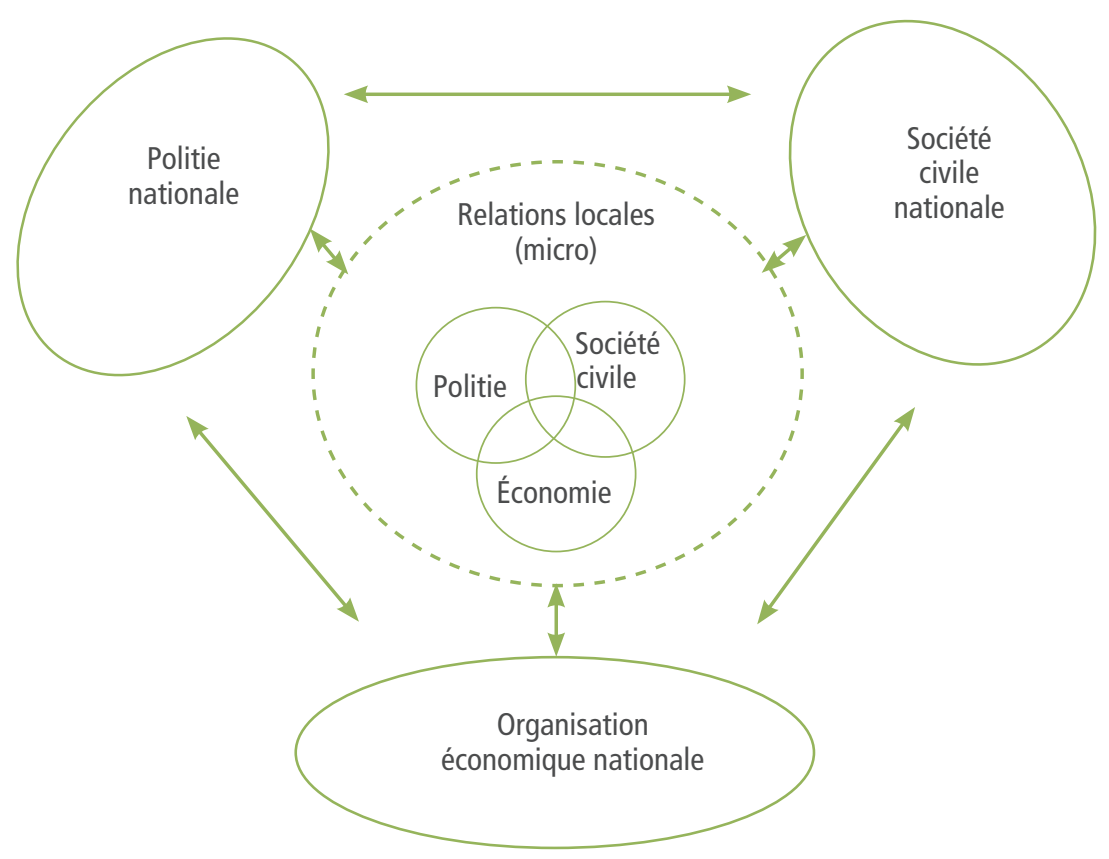

Il faut d'abord prendre en compte les institutions formelles du gouvernement de même que celles de la politie qui y sont rattachées comme les partis politiques. Les éléments que nous désignons comme l'État local (local state) incluent les institutions politiques et gouvernementales à l'échelle locale, qu'elles opèrent ou non de façon autonome. Même lorsque l'État local bénéficie de ses propres ressources et d'une autonomie substantielle, il demeure niché au sein d'un ensemble d'institutions faisant partie de l'État-nation. Les services juridiques, les ressources administratives, fiscales et politiques qui donnent de l'autonomie au gouvernement local émanent de lois et de pratiques institutionnelles d'un pays ou d'une de ses parties. Ces composants peuvent alors contraindre les gouvernements locaux et les autres acteurs du même ordre. Les acteurs situés à des niveaux supérieurs peuvent également jouer un rôle actif en tant qu'agents de gouvernance au niveau local.

Parallèlement à cette dimension verticale des relations avec les échelons supérieurs des hiérarchies étatiques, la littérature montre comment la gouvernance à l'échelle locale lie l'État local à la société. Les travaux américains sur la gouvernance urbaine dénotent le rôle décisif des dynamiques de la relation entre 
la société civile et les autorités locales (Dahl, 1961; Hunter, 1953; Stone, 1989). Ceux menés en Europe relèvent de plus en plus des dynamiques similaires (Pierre, 1999; Sellers, 2002). La plupart des chercheurs se sont penchés sur les dynamiques liées au pouvoir politique sur le plan local, et Stone (1989) stipule que le pouvoir des gouvernements locaux dépend de l'appui et de la mobilisation des autres ressources de la société locale. Les rapports sur la gestion des ressources de propriété commune (Ostrom, 1990) ou sur la gouvernance des écosystèmes (Mazmanian et Kraft, 1999) suggèrent que la participation des organisations et des acteurs locaux peut s'avérer essentielle à la réalisation de la politique.

Les travaux en économie politique relèvent des dynamiques similaires dans les économies de pays en développement et de plus en plus au sein de pays développés. La littérature sur les différents types de capitalismes nationaux dans les pays avancés a décrit un modèle sophistiqué rendant compte de la façon dont les systèmes institutionnels nationaux dépendent non seulement des relations au sein des entreprises (les contrats d'embauche, les relations avec les ouvriers et les décisions d'investissements), mais les façonnent (Hall et Soskice, 2001). Culpepper (2003) montre par exemple comment la coopération locale peut s'avérer essentielle au bon fonctionnement des arrangements capitalistes nationaux dans une économie de marché coordonnée. Les chercheurs ayant soumis les capitalismes nationaux (comme l'Allemagne et les États-Unis) à une étude désagrégée ont relevé la présence de différents arrangements à l'échelle régionale (Herrigel, 1996; Sabel et autre, 1989). Les économies locales englobent également des éléments de l'économie dans la gouvernance des entreprises capitalistes. Les marchés de consommation, ainsi que les acquis d'une communauté tels que le logement et l'immobilier, et les décisions liées à l'emplacement d'une entreprise sont étroitement liés aux caractéristiques physiques des communautés et des régions. Les gouvernements jouent notamment un rôle déterminant en garantissant localement la fourniture de biens publics pour les entreprises en général comme des infrastructures (Crouch, 2004) ou des économies d'échelle rendant l'emplacement rentable pour les entreprises locales.

À l'intersection de ces domaines, la société civile compte une grande variété d'associations et d'organisations sociales partiellement distinctes de la politie et de l'économie. Les professionnels et les groupes communautaires locaux, les associations de quartier et les organisations religieuses appartiennent tous à cette sphère. La société civile semble plus intégrée dans l'organisation des communautés. Or, comme l'avance Skocpol (2003), elle peut également s'organiser au-delà du niveau local pour jouer un rôle à l'échelle nationale. Bien que certaines associations soient essentiellement locales, d'autres comme les partis politiques, les associations professionnelles ou même des groupes activistes en environnement ont également une portée nationale et transnationale.

Les associations de la société civile organisées localement ont avantage à entretenir des liens avec la politie locale en tant que type d'association en soi. Les partis politiques et les organismes communautaires liés à ces associations existent surtout dans le but d'influencer l'État local. Les associations comme les 
organisations de quartier ou les organismes de services publics locaux cherchent également à profiter des décisions politiques locales. Les élus dépendent souvent des partis ou des membres des associations civiques locales pour maintenir leur position au sein de l'administration locale. Les associations civiques telles que les chambres de commerce représentent les intérêts économiques et commerciaux dans la politie tout en pouvant jouer un rôle clé dans le développement de coalitions autour de questions liées au programme politique local.

Malgré ces distinctions, les théories récentes sur les sources du pouvoir et la bonne gouvernance locale dénotent l'importance des relations entre ces secteurs. La majorité de ces relations prennent place au niveau local et régional. Une approche s'appuyant sur ces relations à l'échelle locale et sur leur rôle dans le schéma général des relations État-société relève la présence de plusieurs types de relations institutionnelles dans les modèles locaux des relations État-société. Ces alternatives offrent un outil utile pour classifier les variations transnationales des systèmes nichés au sein de la gouvernance locale.

\section{— LES INFRASTRUCTURES INSTITUTIONNELLES DE LA GOUVERNANCE LOCALE}

Le corpus de connaissances quant aux relations entre les politiques nationales et locales dénote la présence d'interconnexions déterminantes. Au sein de l'État, les politiques nationales sont influencées par les mises en œuvre faites au niveau local. Au sein de la société civile et de l'économie politique, la mobilisation et l'organisation locales sont également déterminantes. Nous devons alors mieux saisir l'ensemble des différentes manières dont se combinent les relations État-société aux différents niveaux.

Notre analyse se concentre sur ces configurations d'institutions que nous nommons les « infrastructures de gouvernance locale » et qui sont tenues pour acquises - un élément central des institutions (Immergut, 1998; March et Olsen, 1984). Audelà des institutions formelles, elles comptent les règlements et les organisations informelles ainsi que les éléments informels des organismes formels (Helmke et Levitsky, 2004). Encore plus pertinent, elles s'étendent aux institutions de la société civile et étatiques. Enfin, ces infrastructures opèrent à l'échelle urbaine, communautaire et régionale, elles institutionnalisent les acteurs, les règles du jeu, les normes, les programmes et les orientations culturelles de la gouvernance locale.

Pour simplifier la question, il convient de s'attarder aux modèles d'un système à deux ordres (le local et le national) et d'étudier ainsi les configurations institutionnelles qui lient l'État local - défini comme les composants de l'État présent au sein des villes - aux éléments étatiques à des niveaux supérieurs et à une plus grande échelle. Ces infrastructures de gouvernance locale comptent des institutions aux deux ordres. À l'échelle locale, elles incluent les institutions formelles du gouvernement local parallèlement aux organisations informelles ou parapubliques faisant partie des outils de gouvernance locale. Bien que certains aspects du gouvernement local soient purement locaux, plusieurs règles du jeu politique 
n'émanent pas du niveau local. Les lois électorales et les systèmes locaux d'administration publique proviennent notamment de la législation nationale et de dispositions constitutionnelles ou sont appuyés par des sources à une plus grande échelle. L'infrastructure de gouvernance locale s'étend également aux fonctions stratégiques exercées par le gouvernement local, aux capacités institutionnelles fiscales et politiques qu'elle possède, aux contraintes auxquelles elle fait face et aux façons qu'elle est supervisée ou régulée. Outre ces paramètres administratifs fiscaux et légaux, elle comprend également les relations de mise en œuvre de politique souvent qualifiées de relations " centrales-locales " (central-local relations) (Ansell et Di Palma, 2004; Tarrow, 1977).

La littérature actuelle sur les institutions nationales au chapitre de la gouvernance locale s'est limitée à ces systèmes d'institutions gouvernementales. En négligeant la structure de la société civile, elle laisse un vide conceptuel. Bien que cette distinction entre la société et l'État soit utile, elle va à l'encontre des données sur le fonctionnement de la société civile. Les systèmes des partis politiques varient considérablement dans leur mode d'organisation et dans la façon qu'ils sont liés aux autres systèmes institutionnels nationaux et aux lois électorales. L'organisation des affaires, la main-d'œuvre et les intérêts économiques varient également d'un pays à l'autre. Des travaux sur la participation civique et le capital social ont mis en évidence les différences nationales dans un autre secteur de la société civile qui mobilise généralement le niveau local pour influencer la politique (Putnam, 2002). Des études sur les mouvements sociaux et la gouvernance urbaine ont démontré que l'organisation au sein de la société peut également influencer les modèles nationaux de gouvernance locale. La façon dont ces variations dans les dynamiques de la société civile s'associent à l'infrastructure gouvernementale de la gouvernance locale n'a toutefois pas été fouillée. Or, ce lien est souvent étroit et va dans les deux sens. L'infrastructure des organisations politiques et civiques influence la réalité opérationnelle de la gouvernance locale et l'évolution des institutions gouvernementales locales. En même temps, l'organisation au sein de la société civile est en partie le produit des possibilités et des ressources offertes par les institutions gouvernementales.

Au sein des démocraties électorales de pays développés, ces infrastructures de gouvernance locale servent des fonctions similaires. Nous pouvons considérer ces fonctions comme des solutions aux logiques descendante (top-down) et ascendante (bottom up) des relations locales-nationales. La logique descendante correspond à la logique du pouvoir infrastructurel et au point de vue des élites nationales. Elle s'inscrit également dans l'autorité hiérarchique formelle propre à la structure légale et administrative de l'État-nation. Dans cette logique, une infrastructure de gouvernance locale devrait générer la mise en œuvre efficace de décisions prises à des échelons supérieurs de l'État-nation par des gouvernements locaux de même que la réalisation des objectifs étatiques jusqu'à la société civile. Analysés sous l'angle de la théorie du mandant-mandataire (principal-agent theory), un gouvernement local et une infrastructure civique fonctionnant efficacement n'ont pas à être sous le contrôle d'une autorité supérieure pour être efficace. Avec une surveillance adéquate, les autorités locales peuvent accomplir la mission de l'État. 
Les travaux sur la gouvernance locale ont également adopté une perspective ascendante dans la définition de ce que devrait être un système efficace de relations nationales-locales. Dans cette perspective, une infrastructure efficace de gouvernance locale offre aux communautés, aux mouvements et aux citoyens un pouvoir local. Ces mécanismes de responsabilisation englobent d'une part les moyens offerts aux communautés pour se gouverner au niveau local et d'autre part les outils fournis aux mouvements, aux groupes et aux citoyens au sein de ces communautés pour exercer leur influence dans les décisions au niveau national. Un point commun qui ressort d'études portant sur la gouvernance des ressources communes (Ostrom, 1990) ou sur la façon dont les régimes urbains ont rassemblé le milieu des affaires et les dirigeants politiques locaux (Stone, 1989) est l'adoption d'une variante de cette première logique. L'influence à un échelon supérieur exige l'agrégation des préférences et des intérêts locaux et une capacité de les représenter dans une prise de décision nationale. Ces processus peuvent se faire à l'aide de partis organisés, de groupes d'intérêts, de mouvements sociaux, voire de représentants, qui viennent soutenir les intérêts de la clientèle qu'ils servent.

Qu'elles soient analysées dans une perspective descendante ou ascendante, les infrastructures de gouvernance locale ne retracent que partiellement la construction et la démocratisation de l'État au niveau national. En plus de la construction d'un État de droit (constitutional state), elles émanent également de la construction de l'État politique (policy state). Avec l'élargissement des politiques de l'État vers le développement économique local, l'offre de services sociaux et la protection de l'environnement, les demandes pour des pouvoirs infrastructurels requièrent un rôle pour la gouvernance locale. En raison de l'incidence de ces politiques et de la participation grandissante d'acteurs locaux, la mobilisation locale devient de plus en plus essentielle.

Plusieurs travaux sur le fédéralisme fiscal ont tenté de réconcilier les logiques descendante et ascendante d'efficacité de même que leurs interrelations (Boadway et Shah, 2009; Oates, 1999). Dans le cadre de notre analyse, nous supposons la présence de ces deux logiques. Les infrastructures alternatives de gouvernance locale offrent des mécanismes institutionnels distincts pour permettre des relations locales-nationales efficaces, qu'elles soient descendantes ou ascendantes.

\section{- LES INFRASTRUCTURES ALTERNATIVES DE GOUVERNANCE LOCALE}

Les infrastructures alternatives de gouvernance locale renvoient à deux dimensions générales. Une première comprend la relation intergouvernementale verticale entre l'État local et les échelons supérieurs, à l'instar de la littérature traitant des relations centrales-locales. À une extrémité du spectre, les gouvernements locaux prennent des décisions et appliquent les politiques. Au moment où ils reçoivent la responsabilité de mettre en œuvre les programmes de l'État, ils obtiennent également les habilités légales, fiscales et administratives pour le faire. Les autorités locales et nationales, de même que leurs politiques, demeurent étroitement intégrées. À l'autre extrémité du spectre, les gouvernements locaux, possédant des capacités minimales, n'assument pas de responsabilités nationales. 
Au lieu d'obtenir du soutien intergouvernemental, ils renforcent leurs capacités à l'aide de relations avec des acteurs non gouvernementaux. La gouvernance locale procède alors indépendamment, voire à contrario, des politiques nationales et dépend de l'appui de la société civile et de l'économie locale.

Une seconde dimension s'attache à l'incorporation de la société civile, politique et économique. D'un côté, l'incorporation sous-tend l'engagement de la société civile ou du moins son organisation en associations ou en organismes. Parmi ces derniers, on compte les partis politiques, les intérêts économiques et des associations de la société civile comme des groupes de bénévoles, des associations de quartier et des organismes culturels ou de services. D'un autre côté, ces associations ou organismes doivent être intégrés au processus de gouvernance. Il s'agit non seulement de mobilisation, mais aussi d'approbation ou d'appui à la société civile de la part des institutions et des acteurs gouvernementaux. Dès lors que l'incorporation est limitée ou faible, la gouvernance demeure une affaire d'élite politique.

La gouvernance multiniveau présente plusieurs modèles des relations entre l'État national et l'échelon local (voir tableau 1). Dans un système entièrement élitiste (coin inférieur gauche), la gouvernance se fait d'" en haut ». Les autorités nationales dictent les actions locales tandis que l'incorporation politique et civique au niveau local est limitée, voire absente. Ce type d'arrangement se trouve surtout au sein de régimes autoritaires et rarement dans les démocraties contemporaines. L'émergence des institutions gouvernementales locales reflète à la fois une plus grande compréhension de l'importance de la gouvernance locale dans le pouvoir infrastructurel et le renforcement de l'autonomie locale. Le développement et la diffusion de l'État politique - un État qui s'appuie sur la poursuite d'objectifs économiques, sociaux et environnementaux - renforcent l'importance des liens locaux. Le local peut même jouer un rôle non négligeable au sein de régimes autoritaires contemporains telle la République populaire de Chine.

\section{TABLEAU 1 : MODÈLES ALTERNATIFS DES RELATIONS CENTRALES-} LOCALES ENTRE L'ÉTAT ET LA SOCIÉTÉ CIVILE

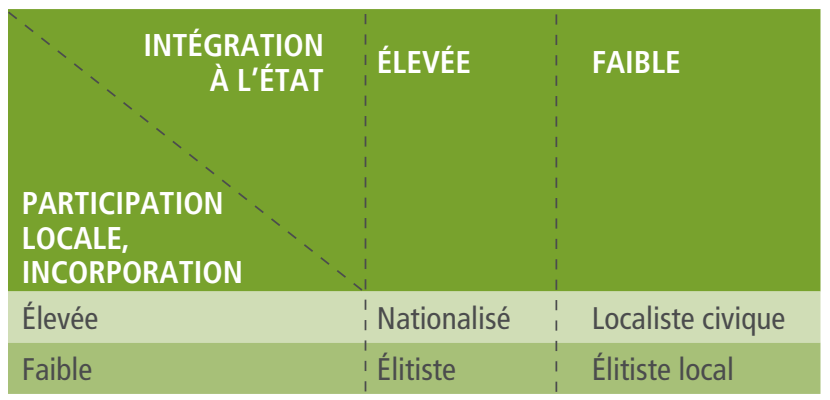


Le tableau 1 indique la présence de plusieurs types de relations entre la société et l'État au sein des systèmes ayant des gouvernements locaux. Dans un milieu " élitiste local ", les autorités locales maintiennent une influence, malgré l'incorporation politique et civique limitée. À l'opposé, l'incorporation politique et civique est très élevée dans le modèle " localiste civique ", alors que l'intégration verticale de l'État local avec la politique nationale reste limitée. À l'intérieur d'un environnement "nationalisé ", les groupes sociaux et politiques à l'échelle locale sont incorporés à l'État local. Les politiques locales de même que l'État local sont néanmoins intégrés à des niveaux étatiques supérieurs.

Ces trois types de relations correspondent à des modèles distincts de gouvernance locale. Chacun favorise un ensemble différent de mécanismes pour l'affirmation du pouvoir infrastructurel par le haut, et la responsabilisation de la communauté par le bas. Ils institutionnalisent divers intérêts en perpétuant les arrangements existants parmi les autorités locales et les relations nationales-locales et les différents modèles organisationnels des partis, des organismes économiques et des associations civiques. Chaque type est issu d'une trajectoire historique précise.

\section{L'infrastructure nationalisée}

Dans l'infrastructure nationalisée, la gouvernance et les politiques locales sont liées aux politiques et aux objectifs nationaux. Les autorités locales mettent en œuvre les politiques formulées à l'échelle nationale. Malgré la présence de problématiques locales, les gouvernements locaux de tout le pays appliquent les mêmes politiques. Au-delà des dispositions gouvernementales, cette infrastructure s'appuie sur un système d'intérêts et de partis nationaux bien organisés (figure 2). Sur le plan national, ces partis et ces organisations représentent les intérêts nationaux au sein des communautés, alors qu'au niveau local, ils intègrent une large part de la population et défendent leurs intérêts au sein des processus décisionnels locaux. Les organisations nationales regroupent également les intérêts locaux, elles les traduisent en programmes pour ensuite les promouvoir à l'échelle nationale et ainsi générer un meilleur soutien localement. De même, en raison de la très grande présence des organisations nationales à l'échelle locale, les politiciens nationaux confient une plus grande autonomie aux autorités locales dans la réalisation locale des objectifs nationaux. 
FIGURE 2: L'INFRASTRUCTURE NATIONALISÉE DE GOUVERNANCE LOCALE

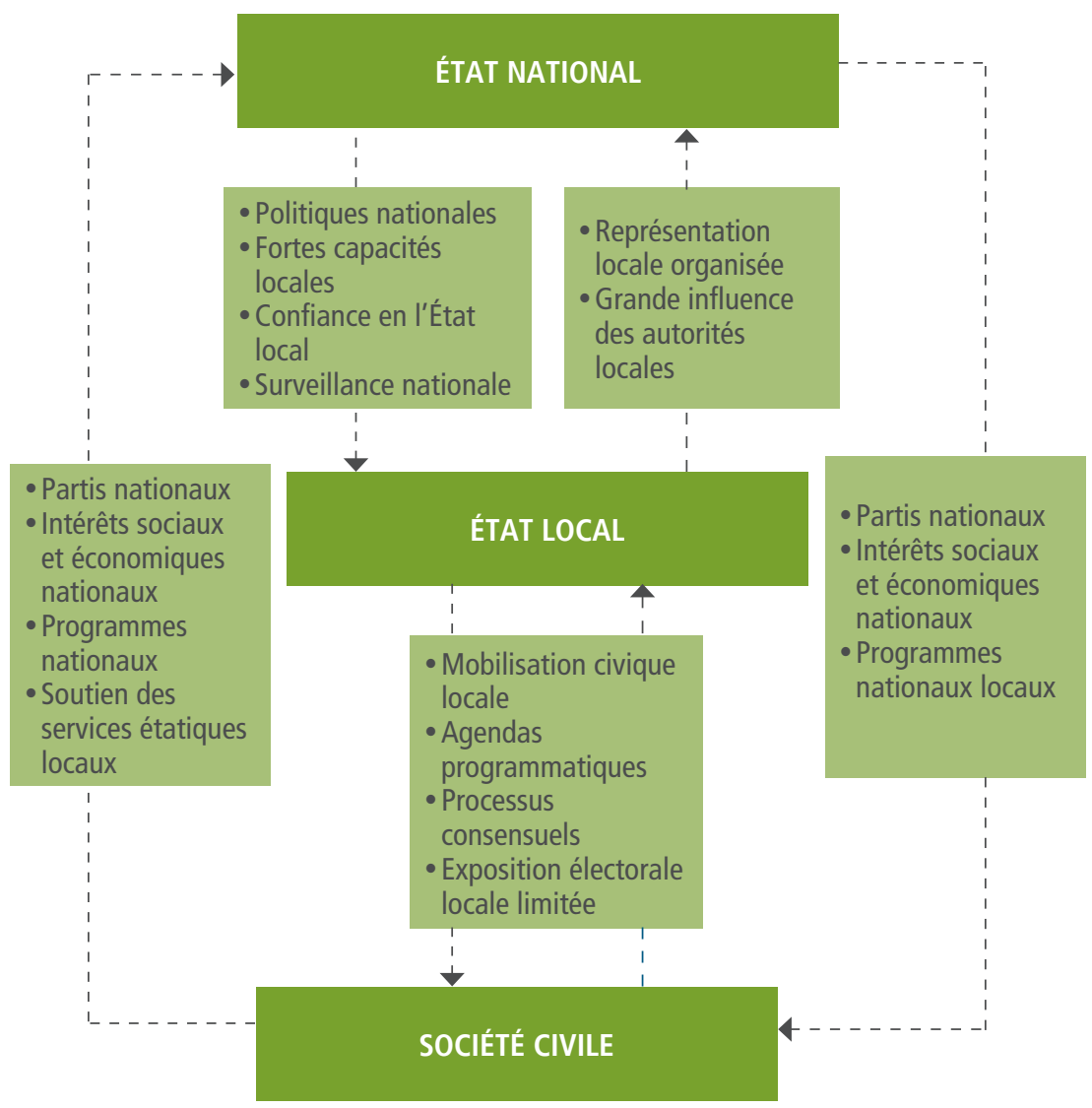

L'infrastructure nationalisée s'inscrit dans la lignée des présomptions wébériennes sur les relations politiques entre les ordres local et national. Ce système associe la fourniture des biens publics aux programmes nationaux et applique les politiques de façon similaire dans toutes les localités du pays. L'organisation de la société civile relève également du national. Les représentants locaux des partis nationaux et des groupes d'intérêts organisés de même que les représentants gouvernementaux des échelons supérieurs coopèrent dans la mise en œuvre des programmes nationaux au niveau local. Ces mêmes organisations représentent les intérêts des localités dans le processus politique national. Cette double fonction nationale et locale - des partis politiques, des groupes d'intérêts organisés et des autorités locales favorisent l'intégration des politiques nationales et locales. 
Cette infrastructure nationalisée s'appuie sur l'intégration organisationnelle des niveaux local et national pour maintenir le pouvoir infrastructurel. Les autorités supérieures fournissent les ressources au niveau local pour la mise en œuvre des politiques tout en supervisant et régulant cette mise en œuvre. Le climat de confiance facilite d'une part la délégation des responsabilités vers les autorités locales et d'autre part l'acceptation des interventions des autorités supérieures au niveau local. Au-delà de l'appareil étatique, les intérêts et les partis organisés hiérarchiquement viennent nourrir cette confiance mutuelle. Ces organisations aident à mobiliser la société civile autour des politiques locales et nationales implantées conjointement par les deux ordres d'autorité. Par ailleurs, elles canalisent la participation civique en faveur de ces politiques tout en veillant à ce que les politiques nationales représentent les intérêts locaux.

La responsabilisation locale sous une infrastructure nationalisée adhère à une logique précise. À l'échelle de l'État, les mécanismes de représentation locale donnent un rôle politique important aux partis organisés nationaux ainsi qu'aux autorités locales. L'infrastructure nationalisée incorpore localement les partis, les entreprises, la main-d'œuvre et les organismes civiques. Cette incorporation demeure néanmoins limitée par l'intégration des institutions nationales et locales. Les institutions participatives locales favorisent les partis nationaux et les systèmes nationaux d'approvisionnement aux mouvements locaux particularisés. Les institutions de gouvernance locale offrent un choix régulier, mais limité aux élections locales et favorisent la formation de coalitions consensuelles entre les partis au lieu d'une gouvernance majoritaire.

Les pays scandinaves et les Pays-Bas correspondent le mieux à ce type de relations locales-nationales. Le cas de la gouvernance locale en Suède est bien documenté. Ses gouvernements locaux figurent parmi les plus habilités des pays développés, et il leur incombe la responsabilité de la gestion de l'État-providence et de différentes fonctions telle la planification urbaine (Sellers et Lidström, 2007). La société civile y est largement organisée et très participative (Rothstein et Stolle, 2003), et les organisations patronales, les syndicats et les organisations civiques jouent généralement un rôle déterminant dans la gouvernance locale (Pierre, 1992 ; Sellers et Kwak, 2011).

Une perspective ascendante vient illustrer comment les origines et le déploiement d'une telle configuration sont en partie le produit de la société civile et des relations locales-centrales. De fortes institutions communautaires ont jeté les bases de l'État moderne. La Scandinavie a développé un appareil étatique national fort au début de l'ère moderne (Knudsen et Rothstein, 1994). Faisant face à des pouvoirs centraux et locaux, les partis, les entreprises et la main-d'œuvre se sont organisés nationalement tout en s'ancrant fermement dans les communautés (Kumlin et Rothstein, 2005). L'État-providence universaliste et égalitaire, une caractéristique déterminante de ce système étatique local, s'est développé à l'échelle nationale à partir des institutions locales de l'époque (Sellers et Lidström, 2007).

Avec la présence d'une infrastructure nationalisée, plusieurs types de logiques relationnelles entre le local et le national reproduisent les éléments essentiels à la structure. Les autorités nationales et locales de même que les associations 
politiques nationales ont avantage à reproduire le système. Lorsqu'ils sont bien implantés au sein d'organisations mobilisées sur la scène locale, les partis nationaux acquièrent la capacité de représenter les communautés locales à l'échelle nationale. Parce que les unités locales des partis et des groupes d'intérêts jouent un rôle prépondérant dans la politique locale, leurs représentants nationaux confient davantage de responsabilités aux autorités locales dans la mise en œuvre de politiques nationales. Les fonctionnaires nationaux ont certes intérêt à superviser les politiques déléguées au niveau local, mais la présence locale d'organismes nationaux accroît leur confiance dans les localités. Avec un service civil nationalisé à l'échelle locale, les autorités locales agissent comme de fervents défenseurs du maintien des pouvoirs locaux. Les services offerts par les gouvernements locaux viennent appuyer les institutions décentralisées et les réformes au sein de l'infrastructure - notamment la consolidation d'entités publiques locales - viennent souvent renforcer les dynamiques de représentation locale.

\section{L'infrastructure localiste civique}

Contrairement à l'infrastructure nationalisée, l'infrastructure localiste civique s'appuie sur une participation généralisée au niveau local avec une intégration étatique et politique minimale aux échelons supérieurs. D'importantes responsabilités sont conférées aux autorités locales qui exercent des capacités et des pouvoirs limités et qui s'appuient sur la société locale pour générer les ressources politiques. Le programme de gouvernance locale est fixé non pas par les intérêts et les partis organisés au niveau national, mais par les associations civiques des communautés. Contrairement à la représentation nationale équilibrée et la gouvernance locale consensuelle générée par l'infrastructure nationalisée, l'infrastructure localiste civique génère une meilleure écoute locale des groupes les plus puissants et les plus mobilisés. Les autorités locales peuvent également travailler dans le sens contraire à la gouvernance nationale.

Au sein de cette infrastructure, les politiques mises en œuvre localement relèvent d'initiatives des autorités locales. Les règles du jeu politique encouragent la réactivité de la société civile locale. Il n'y a pas de représentation fiable et constante des intérêts nationaux au sein des localités par les autorités locales, les associations civiques nationales, les partis nationaux ou les groupes d'intérêts organisés. Le traitement réservé aux questions locales aux échelons supérieurs dépend de la mouvance des coalitions d'intérêts en jeu et il n'y a pas de lien constant entre les niveaux local et national. La confiance intergouvernementale qui pourrait poser les jalons d'une éventuelle délégation de pouvoir aux autorités locales reste limitée (figure 3). 

LOCALE

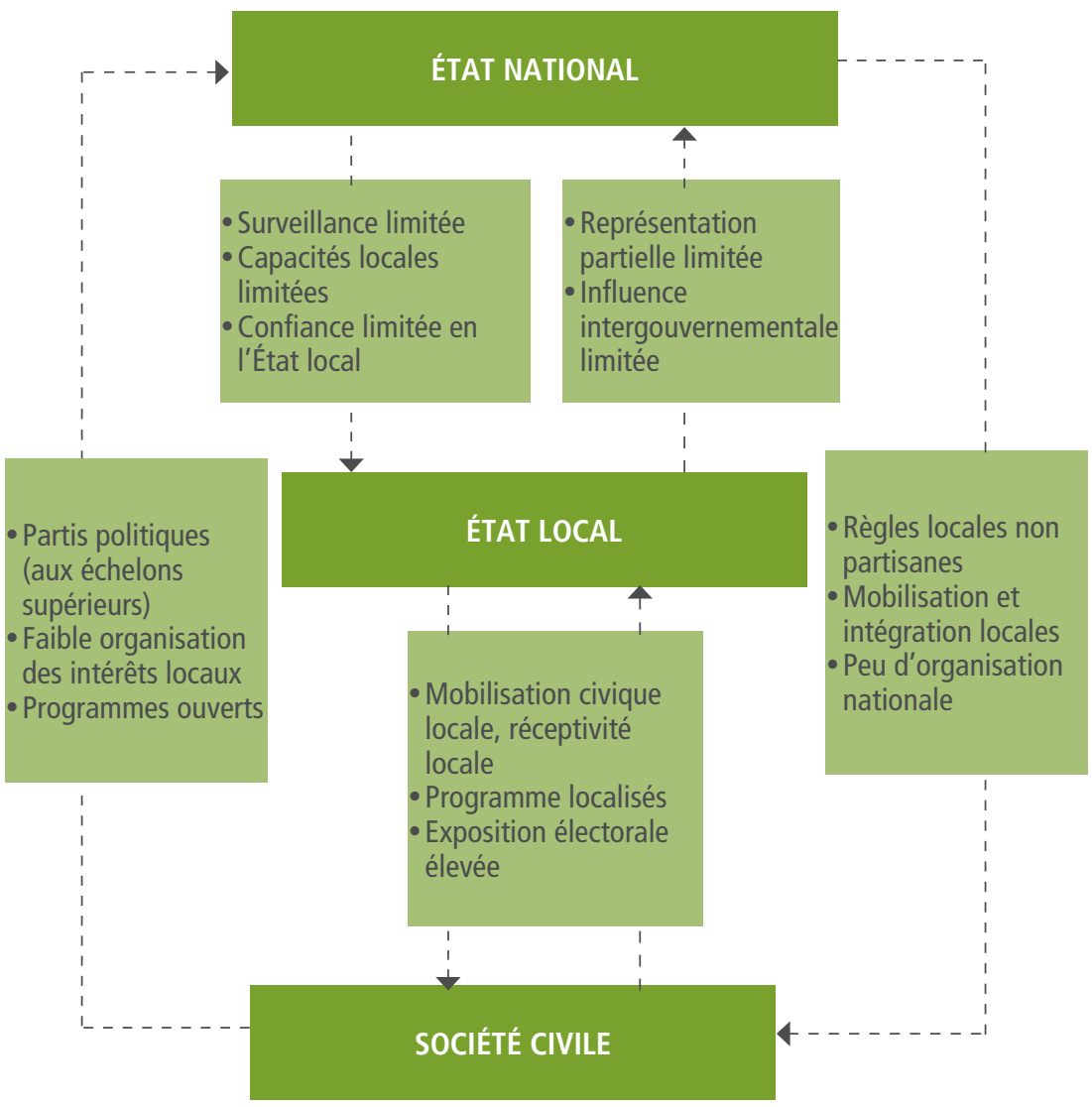

Au sein d'une pareille infrastructure, le pouvoir est généralement distribué entre les localités et les communautés. L'infrastructure localiste civique s'appuie sur l'exercice de l'autorité locale au détriment d'une intégration des autorités locales dans la politique aux échelons supérieurs. Les mécanismes infrastructurels mettent en place un régime de responsabilités à l'égard de la société locale ou limite les capacités locales en évitant ainsi la supervision par une autorité d'un échelon supérieur. La société civile participe et s'intègre au niveau local par l'entremise des associations locales, alors que les partis politiques et les intérêts organisés aux échelons supérieurs entretiennent peu de relations avec les politiques locales. Lorsque les autorités nationales souhaitent intervenir localement, leurs interventions restent limitées à un secteur fonctionnel précis. Le peu de confiance entre les ordres de gouvernement engendre des formes limitées de coordination. 
Dans un tel système, la responsabilisation se fait d'abord au niveau local à travers les coalitions ou les organisations plus ou moins partisanes. La participation locale est élevée alors que les groupes d'intérêts exercent une influence décisive. Aux échelons supérieurs, les partis et les groupes d'intérêts organisés sont peu enracinés dans les formations politiques locales et rassemblent les intérêts locaux indépendamment de la gouvernance locale. Les représentants institutionnels des autorités locales n'ont pas accès à des mécanismes établis de représentation aux échelons supérieurs. L'influence et les intérêts locaux se font plutôt à l'aide d'autres canaux comme la présence d'un représentant de district dans la législature nationale ou des mouvements organisés nationalement.

Tout comme les infrastructures nationalisées, les institutions localistes civiques sont nées au sein de sociétés ayant une forte participation politique et civique institutionnalisée. Les pays illustrant le mieux ce type d'arrangement sont les nations qui ont transposé l'héritage britannique aux nouvelles colonies. Les communautés de propriétaires fonciers blancs ont mis en place les fondations de l'infrastructure institutionnelle. Les territoires inhospitaliers et coûteux encourageaient des formes locales d'administration qui offraient plus d'autonomie aux communautés locales. Les formes fragmentées d'États centraux fédérés que sont l'Australie, le Canada et les États-Unis émanent d'influences similaires. Tout au long du développement de l'appareil étatique, la fragmentation institutionnelle aux échelons supérieurs a miné la croissance et l'influence des partis politiques nationaux, des groupes d'intérêts et des structures intergouvernementales nationales telle la fonction publique (Silberman, 1993; Skowronek, 1982). Comme Tocqueville l'a observé aux ÉtatsUnis, les autorités locales et la société civile ont fondé la gouvernance territoriale.

L'infrastructure de la gouvernance locale dans les régions urbaines des ÉtatsUnis illustre ces conditions. L'analyse comparative de cas transatlantiques montre comment les autorités locales de villes américaines opèrent plus indépendamment des autorités centrales et sont davantage soumises au milieu des affaires et des organisations civiques par rapport à l'Europe (Savitch, Kantor et Vicari, 2002; Sellers, 2002). Sellers relève notamment, dans une étude axée sur trois villes aux États-Unis, la présence d'une gouvernance locale dominée par une structure ouverte avec différentes formes de mobilisation civique et de coalition et tributaire des ressources politiques et fiscales des communautés locales. Des comparaisons effectuées à l'aide de sondages sur le pouvoir et l'influence dans la gouvernance locale aux États-Unis confirment ces tendances (Sellers, 2007).

Cette infrastructure devrait générer de nouvelles dynamiques. En l'absence d'intérêts locaux bien organisés aux échelons supérieurs, l'autonomisation du niveau local s'appuie sur des dispositions assurant la réceptivité locale. Parce qu'ils ne croient pas qu'un système national de partis politiques locaux puisse s'immiscer dans les politiques locales, les politiciens nationaux tentent de sécuriser l'imputabilité par la limitation des compétences locales ou les exigences en termes de participation civique. Les associations civiques apportent un soutien additionnel aux institutions qui viennent renforcer leur rôle et celui des électeurs au détriment des partis politiques et des groupes d'intérêts. Avec une capacité locale moins grande et moins de pouvoir que les systèmes nationalisés, les autorités locales 
n'arrivent pas à contrecarrer les menaces posées par les échelons supérieurs. En fournissant moins de services, les gouvernements locaux ont moins d'appui populaire. Ces dynamiques aident à perpétuer la séparation institutionnelle entre les niveaux national et local de même que la dépendance de l'infrastructure à la participation locale.

\section{L'infrastructure élitiste locale}

La troisième forme d'infrastructure affiche une participation limitée des organisations politiques au niveau local. Les élites au sein des gouvernements locaux dominent au détriment des partis nationaux, des groupes d'intérêts organisés nationalement et des autres types d'associations. Les systèmes électoraux majoritaires locaux et la fragmentation géopolitique locale viennent renforcer la position dominante de l'élite locale par rapport aux citoyens et aux influences organisationnelles plus larges. Outre au sein de réseaux clientélistes, ou pour les élites locales ayant gagné une légitimité, la confiance des citoyens entre eux et envers l'État local demeure limitée.

À l'instar de l'infrastructure nationalisée, la législation nationale veille à la prestation des biens publics aux échelles locale et nationale. Par contre, ici les autorités de plus haut niveau supplantent les autorités locales dans la prestation de biens publics et l'élaboration de politiques locales et maintiennent une bonne surveillance. Les autorités locales ont de faibles capacités et elles sont organisées hiérarchiquement. Comme dans l'infrastructure localiste civique, la confiance que portent les autorités nationales aux autorités locales reste faible. Les élites gouvernementales locales de même que les hiérarchies intra-étatiques incarnent le principal mécanisme d'intégration entre le national et le local. Contrairement aux groupes d'intérêts et aux partis politiques nationaux fortement organisés d'une infrastructure nationalisée, les initiatives nationales dépendent de l'influence locale et nationale des élites locales. Les réseaux verticaux des relations entre les élites locales et les représentants de l'État fournissent des possibilités de représentation des intérêts locaux aux échelons supérieurs du gouvernement de même que l'intégration entre les gouvernements local et national.

L'infrastructure combine alors une centralisation légale et administrative à une décentralisation politique (Page et Goldsmith, 1989) et une démobilisation civique. Contrairement à l'infrastructure localiste civique, les autorités locales maintiennent des réseaux denses avec les autorités nationales, et contrairement à l'infrastructure nationalisée dans laquelle les partis politiques et la fonction publique sont hautement organisés, les liens prennent ici la forme de réseaux personnels ou organisés de façon informelle. En l'absence d'une participation citoyenne généralisée (comme dans les deux autres infrastructures), les liens individuels qui unissent les élites officielles et les groupes sociétaux prennent plus d'importance (figure 4). 


\section{FIGURE 4: L'INFRASTRUCTURE ÉLITISTE LOCALE DE GOUVERNANCE LOCALE}

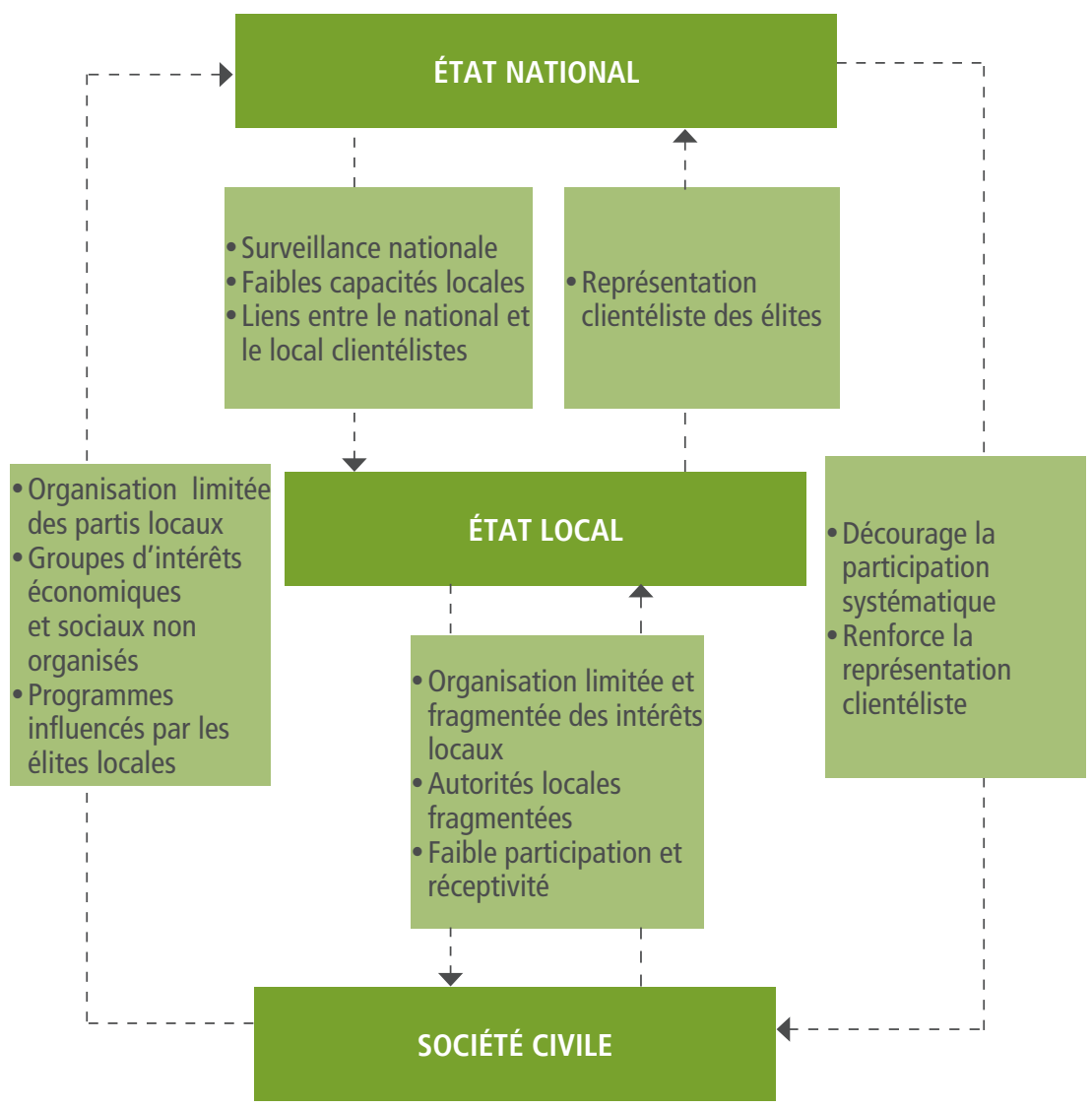

Dans une infrastructure élitiste locale, le pouvoir s'exerce selon les termes mis de l'avant par Mann et plusieurs autres auteurs s'intéressant à l'État. La gouvernance locale française, même avec l'avènement de la décentralisation des années 1980, témoigne du fonctionnement de cette infrastructure (Pinson, 2009; Savitch, Kantor et Vicati, 2002; Sellers, 2002). Les hiérarchies étatiques incarnent le principal outil mis à la disposition des élites politiques nationales souhaitant obtenir des résultats à l'échelle locale. Les autorités hiérarchiques qui mettent en œuvre les politiques locales, les dynamiques de négociation et de réciprocité entre les élites locales et nationales de même que les réseaux verticaux de relations entre les différents niveaux sont des exemples de ce type de mécanisme. La confiance limitée des échelons gouvernementaux plus élevés dans les autorités locales renforce la tendance à s'appuyer sur les autorités nationales pour mettre en œuvre les politiques. Au sein de la société locale, la participation et l'incorporation 
civiques demeurent faibles (Sellers, 2002). Des niveaux d'organisation sociétale moins élevés peuvent limiter l'opposition effective aux initiatives politiques tout en astreignant la mobilisation du soutien pour ces dernières. L'efficacité des actions de l'État local dépend des ressources avancées par les autorités supérieures, des réseaux de relations informelles ou clientélistes entre le gouvernement local et les élites locales et de la capacité des élites gouvernementales locales d'établir leur légitimité politique au sein des communautés.

Malgré la faible intégration civique dans l'infrastructure élitiste locale, elle fournit des mécanismes d'autonomisation locale. La réciprocité dans les réseaux d'élites locales et nationales permet aux élites locales d'obtenir des bénéfices pour leur communauté de façon collective ou individuelle. Les représentants des organes nationaux de surveillance et d'administration, comme les préfets en France, servent partiellement à communiquer et à représenter les intérêts locaux au sein de l'appareil étatique. La façon dont les autorités locales représentent les intérêts de la société locale influence l'autonomisation de la communauté, et les limites à l'incorporation civique peuvent nuire à cette autonomisation. Or, la faible incorporation n'empêche pas l'influence des mouvements locaux aux ordres national et local. Au sein des infrastructures élitistes locales telles que la France, de grandes protestations informelles contre les élites locales ou nationales sont fréquentes (Sellers, 1995).

L'origine des configurations élitistes locales et leurs dynamiques de reproduction diffèrent des autres configurations. Avant l'avènement de la démocratie, une paysannerie subalterne dans les campagnes et une classe moyenne affaiblie dans les villes génèrent des conditions favorables à la création d'un État pour des institutions élitistes locales (Tilly, 1992). Avec l'établissement d'États constitutionnels et démocratiques et une incorporation civique et politique moins soutenue, les élites politiques et administratives locales émanant des gouvernements local et national élaborent l'État politique.

Ce faisant, ces réseaux d'élites perpétuent leur position centrale. Les élites locales s'appuient sur leur base de soutien dans les communautés et sur la prestation de services étatiques par les échelons supérieurs pour maintenir ce soutien. Leur pouvoir au niveau national leur permet de maintenir l'infrastructure institutionnelle locale qui solidifie leur position du gouvernement local tout en minimisant les défis par des élections ou des conditions de participation. Parce qu'elles n'ont pas la confiance des fonctionnaires nationaux pour mettre en œuvre les politiques, le pouvoir des autorités locales est limité parallèlement à une surveillance accrue de la politique locale par les échelons supérieurs. La position prépondérante des élites locales aux niveaux national et local renforce néanmoins la convergence des intérêts entre les fonctionnaires nationaux et locaux, ce qui vient reproduire les éléments essentiels de l'infrastructure élitiste locale (figure 4).

Ces modèles correspondent à des cultures distinctes et mettent en lumière différentes relations, certaines entre l'État et la société et d'autres entre les gouvernements. Chaque modèle répond différemment aux demandes de représentation politique locale, à la politique locale, à l'implantation des politiques de même qu'à la participation locale associée aux démocraties industrielles avancées. Chaque 
infrastructure présente des tensions et des complémentarités institutionnelles qui lui sont propres. Tous possèdent des forces et des faiblesses en tant que modèle pour lier la gouvernance démocratique locale au national.

\section{- CONCLUSION}

L'analyse de ces modèles illustre comment les institutions locales et les relations entre l'État et l'échelon local définissent la décentralisation dans un système multiniveau associé aux sociétés industrielles avancées (tableau 2). Les différences entre les pays pourraient correspondre largement à d'autres modèles déjà abondamment abordés dans les études comparatives des dispositifs institutionnels nationaux tels que les variétés de capitalisme (Hall et Soskice, 2001). Or, l'interaction entre le local et le national que la statique comparative montre englobe des dimensions d'économie politique nationale importantes pour la politique, mais absentes des autres études.

\section{TABLEAU 2 : LES CARACTÉRISTIQUES DES TROIS MODÈLES D'INFRASTRUCTURES ÉTUDIÉS}

\begin{tabular}{|c|c|c|c|}
\hline & $\begin{array}{l}\text { INFRASTRUCTURE } \\
\text { NATIONALISÉE }\end{array}$ & $\begin{array}{l}\text { INFRASTRUCTURE } \\
\text { LOCALISTE } \\
\text { CIVIQUE }\end{array}$ & $\begin{array}{l}\text { INFRASTRUCTURE } \\
\text { ÉLITISTE LOCALE }\end{array}$ \\
\hline $\begin{array}{l}\text { Gouvernement } \\
\text { national }\end{array}$ & $\begin{array}{l}\text { Autonomise tout } \\
\text { en supervisant les } \\
\text { localités }\end{array}$ & $\begin{array}{l}\text { N'autonomise pas et } \\
\text { ne supervise pas les } \\
\text { localités }\end{array}$ & $\begin{array}{l}\text { Supervise sans } \\
\text { autonomiser les } \\
\text { localités }\end{array}$ \\
\hline $\begin{array}{l}\text { Gouvernements } \\
\text { locaux }\end{array}$ & Consensuels & $\begin{array}{l}\text { Représentent la } \\
\text { société civile locale }\end{array}$ & Élites locales \\
\hline Partis politiques & Forts & $\begin{array}{l}\text { Faibles ou } \\
\text { inexistants }\end{array}$ & $\begin{array}{l}\text { Réseaux de l'élite } \\
\text { locale }\end{array}$ \\
\hline $\begin{array}{l}\text { Intérêts } \\
\text { économiques }\end{array}$ & $\begin{array}{l}\text { Représentation } \\
\text { fonctionnelle, } \\
\text { nationale }\end{array}$ & $\begin{array}{l}\text { Représentation des } \\
\text { intérêts civiques et } \\
\text { commerciaux }\end{array}$ & $\begin{array}{l}\text { Groupes élitistes } \\
\text { locaux }\end{array}$ \\
\hline Société civile & $\begin{array}{l}\text { Mobilisée, organisée } \\
\text { nationalement }\end{array}$ & $\begin{array}{l}\text { Intérêts locaux } \\
\text { mobilisés }\end{array}$ & $\begin{array}{l}\text { Peu mobilisée, } \\
\text { intérêts restreints }\end{array}$ \\
\hline $\begin{array}{l}\text { Représentation } \\
\text { des intérêts } \\
\text { intergouverne- } \\
\text { mentaux local- } \\
\text { national }\end{array}$ & $\begin{array}{l}\text { Gouvernements } \\
\text { locaux, } \\
\text { administrateurs, } \\
\text { représentation } \\
\text { collective }\end{array}$ & $\begin{array}{l}\text { Gouvernements } \\
\text { représentent les } \\
\text { intérêts civiques et } \\
\text { commerciaux }\end{array}$ & $\begin{array}{l}\text { Élites } \\
\text { gouvernementales } \\
\text { locales }\end{array}$ \\
\hline $\begin{array}{l}\text { Intérêts non } \\
\text { gouvernementaux } \\
\text { dans les institutions }\end{array}$ & $\begin{array}{l}\text { Partis politiques, } \\
\text { organisations } \\
\text { patronales et } \\
\text { syndicats organisés } \\
\text { nationalement }\end{array}$ & $\begin{array}{l}\text { Intérêts } \\
\text { commerciaux, } \\
\text { groupes civiques }\end{array}$ & $\begin{array}{l}\text { Limités aux réseaux } \\
\text { de clients }\end{array}$ \\
\hline
\end{tabular}


Les modèles révèlent également les limites de la convergence prédites par les analystes dans les infrastructures de gouvernance multiniveau. Les pays développés, et d'autres aussi, ont convergé vers ces recommandations sur la littérature fiscale fédéraliste en tant que fournisseurs de biens publics locaux (Boadway et Shah, 2009). Les routes locales et la planification urbaine locale sont notamment généralement attribuées aux gouvernements locaux dans les trois types d'infrastructures. Les tendances générales vers une gouvernance " néolibérale " introduisent des réformes telles que la privatisation et la décentralisation (Peck et Tickell, 2002). La compétition internationale croissante entre les localités génère une augmentation de la recherche locale d'entreprises et de travailleurs qualifiés (Savitch, Kantor et Vicati, 2002). Avec la généralisation des mouvements transnationaux à travers les sociétés industrielles, les autorités locales tentent de les intégrer dans le processus d'élaboration des politiques de façon partiellement convergente (Sellers, 2002).

Malgré ces influences, diverses logiques reproduisent des infrastructures institutionnelles particulières pour la gouvernance locale. Ces variations nationales dépendent non seulement des différences au niveau national - question abordée par les études comparatives des institutions politiques -, mais également des relations entre les niveaux des trois modèles et du type d'influence qu'ils génèrent.

\section{BIBLIOGRAPHIE}

Ansell, C. K. et G. Di Palma (dir.) (2004). Restructuring Territoriality: Europe and the United States Compared, Cambridge, Cambridge University Press.

Boadway, R. W. et A. Shah (2009). Fiscal Federalism: Principles and Practices of Multiorder Governance, New York, Cambridge University Press.

Crouch, C. (2004). Changing Governance of Local Economies: Responses of European Local Production Systems, Oxford, Oxford University Press.

Culpepper, P. D. (2003). Creating Cooperation: How States Develop Human Capital in Europe, Ithaca, Cornell University Press.

Dahl, R. A. (1961). Who Governs? Democracy and Power in an American City, New Haven, Yale University Press.

Hall, P. A. et D. W. Soskice (2001). Varieties of Capitalism the Institutional Foundations of Comparative Advantage, Oxford, Oxford University Press.

Helmke, G. et S. Levitsky (2004). "Informal Institutions and Comparative Politics: A Research Agenda ", Perspectives on Politics, vol. 2, n 4, p. 725-740.

Herrigel, G. (1996). Industrial Constructions: The Sources of German Industrial Power, Cambridge, Cambridge University Press.

Hesse, J. J. (1991). Local Government and Urban Affairs in International Perspective: Analyses of Twenty Western Industrialised Countries, Baden-Baden, Nomos.

Hunter, F. (1953). Community Power Structure: A Study of Decision Makers, Chapel Hill, University of North Carolina Press.

Immergut, E. M. (1998). « The Theoretical Core of the New Institutionalism », Politics \& Society, vol. 26, n 1 , p. 5-34. 
John, P. et A. Cole (2000). "When Do Institutions, Policy Sectors, and Cities Matter? ", Comparative Political Studies, vol. 33, n² 2, p. 248-268.

Knudsen, T. et B. Rothstein (1994). "State Building in Scandinavia », Comparative Politics, vol. 26, n 2, p. 203-220.

Kumlin, S. et B. Rothstein (2005). " Making and Breaking Social Capital ", Comparative Political Studies, vol. 38, n 4, p. 339-365.

Lidström, A. (2003). Kommunsystem i Europa, Malmö, Liber.

Mann, M. (1984). "The Autonomous Power of the State: Its Origins, Mechanisms and Results ", European Journal of Sociology / Archives européennes de sociologie, vol. 25, $\mathrm{n}^{\circ} 2$, p. 185-213.

March, J. G. et J. P. Olsen (1984). « The New Institutionalism: Organizational Factors in Political Life ", The American Political Science Review, vol. 78, n 3, p. 734-749.

Mazmanian, D. A. et M. E. Kraft (1999). Toward Sustainable Communities: Transition and Transformations in Environmental Policy, Cambridge, MIT Press.

Nordstrom, B. J. (2000). Scandinavia since 1500, Minneapolis, University of Minnesota Press.

Oates, W. E. (1999). "An Essay on Fiscal Federalism ", Journal of Economic Literature, vol. $37, n^{\circ} 3$, p. 1120-1149.

Ostrom, E. (1990). Governing the Commons: The Evolution of Institutions for Collective Action, Cambridge, Cambridge University Press.

Page, E. et M. Goldsmith (1989). Central and Local Government Relations: A Comparative Analysis of West European Unitary States, London, Sage Publications.

Peck, J. et A. Tickell (2002). "Neoliberalizing Space ", Antipode, vol. 34, n 3, p. 380-404.

Pierre, J. (1999). " Models of Urban Governance ", Urban Affairs Review, vol. 34, n 3, p. 372-396.

Pierre, J. (1992). « Organized Capital and Local Politics: Local Business Organizations, Public-Private Committees, and Local Government in Sweden ", Urban Affairs Review, vol. 28, n², p. 236-257.

Pinson, G. (2009). Gouverner la ville par projet, Paris, Presses de Sciences Po.

Putnam, R. (2002). Democracies in Flux: The Evolution of Social Capital in Contemporary Society, New York, Oxford University Press.

Putnam, R., R. Leonardi et R. Nanetti (1993). Making Democracy Work: Civic Traditions in Modern Italy, Princeton, Princeton University Press.

Rothstein, B. et D. Stolle (2003). "Introduction: Social Capital in Scandinavia ", Scandinavian Political Studies, vol. 26, $n^{\circ}$ 1, p. 1-26.

Sabel, C. F. et autres (1989). "Regional Prosperities Compared: Massachusetts and Baden-Württemberg in the 1980s ", Economy and Society, vol. 18, n 4, p. 374-404.

Savitch, H. V., P. Kantor et S. H. Vicari (2002). Cities in the International Marketplace: The Political Economy of Urban Development in North America and Western Europe, Princeton, Princeton University Press.

Schickler, E. (2001). Disjointed Pluralism: Institutional Innovation and the Development of the U.S. Congress, Princeton, Princeton University Press. 
Sellers, J. M. (2007). "Unions and the Strategic Context of Local Governance ", dans L. Turner et D. B. Cornfeld (dir.), Labor in the New Urban Battlegrounds, Ithaca, Cornell University Press, p. 35-52.

Sellers, J. M. (2005). " Re-Placing the Nation », Urban Affairs Review, vol. 40, n 4, p. 419-445.

Sellers, J. M. (2002). Governing from Below: Urban Regions and the Global Economy, New York, Cambridge University Press.

Sellers, J. M. (1995). "Litigation as a Local Political Resource: Courts in Controversies over Land Use in France, Germany, and the United States ", Law and Society Review, vol. $29, n^{\circ} 3$, p. 475-516.

Sellers, J. M. et S.-Y. Kwak (2011). «State and Society in Local Governance: Lessons from a Multilevel Comparison ", International Journal of Urban and Regional Research, vol. 35, $\mathrm{n}^{\circ} 3$, p. 620-643.

Sellers, J. M. et A. Lidström (2007). " Decentralization, Local Government, and the Welfare State ", Governance, vol. 20, n 4, p. 609-632.

Sellers, J. M., A. Lidström et Y. Bae (à paraître). Local Linkages and Governance in Democratic Societies: A Global Comparison.

Silberman, B. S. (1993). Cages of Reason: The Rise of the Rational State in France, Japan, the United States, and Great Britain, Chicago, University of Chicago Press.

Skocpol, T. (2003). Diminished Democracy: From Membership to Management in American Civic Life, University of Oklahoma Press.

Skowronek, S. (1982). Building a New American State: The Expansion of National administrative Capacities, 1877-1920, Cambridge, Cambridge University Press.

Soifer, H. (2008). " State Infrastructural Power: Approaches to Conceptualization and Measurement ", Studies in Comparative International Development, vol. 43, n 3, p. 231-251.

Stone, C. N. (1989). Regime Politics: Governing Atlanta, 1946-1988, Lawrence, University Press of Kansas.

Tarrow, S. G. (2005). The New Transnational Activism, Cambridge, Cambridge University Press.

Tarrow, S. G. (1977). Between Center and Periphery: Grassroots Politicians in Italy and France, New Haven, Yale University Press.

Thelen, K. A. (2004). How Institutions Evolve: The Political Economy of Skills in Germany, Britain, the United States, and Japan, Cambridge, Cambridge University Press.

Tilly, C. (1992). Coercion, Capital, and European States, AD 990-1992, Cambridge, Blackwell.

Varshney, A. (2002). Ethnic Conflict and Civic Life: Hindus and Muslims in India, New Haven, Yale University Press.

Vetter, A. (2007). Local Politics: A Resource for Democracy in Western Europe? Local Autonomy, Local Integrative Capacity, and Citizens' Attitudes Toward Politics, Lanham, Lexington Books. 